\title{
A Unilateral Variation in Triceps Brachii Muscle Demonstrating a Fourth Head
}

\author{
Shveta Swami ${ }^{1}$ Virendra Budhiraja ${ }^{1}$ Deepak Sharma² Rimpi Gupta ${ }^{1}$ Swati Bansal' \\ 1Department of Anatomy, Kalpana Chawla Government Medical \\ College, Karnal, Haryana, India \\ ${ }^{2}$ Department of Pediatric and Preventive Dentistry, R. P. Welltar

\begin{abstract}
Address for correspondence Shveta Swami, MBBS, MS, Department of Anatomy, Kalpana Chawla Government Medical College, Karnal, Haryana 132001, India (e-mail: shveta_swami@rediffmail.com).
\end{abstract} Hospital, Karnal, Haryana, India

Ann Natl Acad Med Sci (India) 2021;57:244-246.

\begin{abstract}
Introduction Triceps brachii muscle is the only muscle of posterior compartment of arm, consisting of three heads-long, lateral, and medial. Radial nerve and profunda brachii artery run in the radial groove that separate lateral and medial head. Evolutionarily, triceps has many subheads which either fused or disappeared. Therefore, the knowledge of muscle is essential anthropologically and clinically, and this study aims to study the anatomical variations of triceps brachii muscle.

Case Report In the present case, during routine dissections of undergraduate MBBS students, a fourth head of origin of triceps brachii muscle was seen in a male cadaver in the right arm. The variation was seen only unilaterally in cadaver. The origin was tendinous arising from the posteromedial aspect of upper part of the shaft of the humerus

Keywords

- triceps brachii

- variations

- fourth head

- neurovascular bundle

- compression syndrome close to the surgical neck above the radial groove. This tendon was arching over the neurovascular bundle containing radial nerve and profunda brachii artery.

Discussion and Conclusion The variations of triceps brachii muscles are mentioned in literature but are uncommon and if tendinous fourth head is present over the neurovascular bundles, it may lead to compression syndrome. Hence, these variations are of great importance to the radiologists, surgeons, and orthopaedicians while dealing with posterior compartment of arm.
\end{abstract}

\section{Introduction}

The triceps brachii muscle belongs to the posterior compartment of the arm. It has medial, long, and lateral heads and is an extensor of the forearm at the elbow joint. The long head arises by a flattened tendon from the infraglenoid tubercle of the scapula, blending above with the glenohumeral capsule. The lateral head arises by a flattened tendon from a narrow, linear, oblique ridge on the posterior surface of the humeral shaft, and from the lateral intermuscular septum. The medial head, which is overlapped posteriorly by the lateral and long heads, has a particularly extensive origin. It is attached to the entire posterior surface of the humeral shaft, below the radial groove at the medial border of the humerus; the medial intermuscular septum; and the lower part of the lateral intermuscular septum. ${ }^{1}$

The variant triceps muscle is clinically important for surgeons dealing with entrapment or compressive neuropathies, orthopaedicians operating on fractures of the humerus, anesthetists performing pain management therapies on the upper limb, and radiologists doing radiodiagnostic procedures, for example, computed tomography (CT) scan, magnetic resonance imaging (MRI) of the arm and angiographic studies, and physiotherapists doing electromyography for evaluating and recording electric activity produced by the skeletal muscles. published online

October 26, 2021
DOI https://doi.org/

10.1055/s-0041-1739033

ISSN 0379-038X (c) 2021. National Academy of Medical Sciences (India).

This is an open access article published by Thieme under the terms of the Creative Commons Attribution-NonDerivative-NonCommercial-License, permitting copying and reproduction so long as the original work is given appropriate credit. Contents may not be used for commercial purposes, or adapted, remixed, transformed or built upon. (https://creativecommons.org/licenses/by-nc-nd/4.0/).

Thieme Medical and Scientific Publishers Pvt. Ltd. A-12, 2nd Floor, Sector 2, Noida-201301 UP, India 


\section{Materials and Methods}

During routine dissections of undergraduate students at Kalpana Chawala Govt. Medical College of Karnal in Haryana, a unilateral variation in the number of heads of triceps brachii was found in a male cadaver, aged 60 years, on his right side.

The variation found was fourth head of origin of triceps brachii muscle seen in a male cadaver in the right arm. The origin was tendinous arising from the posteromedial aspect of upper part of the shaft of the humerus close to the surgical neck above the radial groove. This tendon was arching over the neurovascular bundle containing radial nerve and profunda brachii artery. In the lower one-third of the posterior aspect of the arm, the tendon continued as muscular belly which merged with the medial part of long head of triceps brachii. No abnormality was seen in the insertion of triceps brachii. The extra fourth head received its nerve supply through a separate branch from radial nerve ( - Figs. 1 and $\mathbf{2}$ ).

\section{Discussion}

The triceps brachii muscle is normally composed of three heads. A fourth head of triceps may arise from various points on the humerus, scapula, shoulder joint capsule, or the coracoid process. ${ }^{2}$ In the present case, the origin of fourth head of triceps brachii was tendinous arising from the posteromedial aspect of upper part of the shaft of the humerus close to the surgical neck above the radial groove.

Fabrizio and Clemente also demonstrated the fourth head of triceps brachii arising as a single tendon from the proximal posteromedial aspect of the shaft of humerus and distal to the shoulder capsule. The tendon of this fourth head passed along the medial aspect of the humerus and formed a muscle

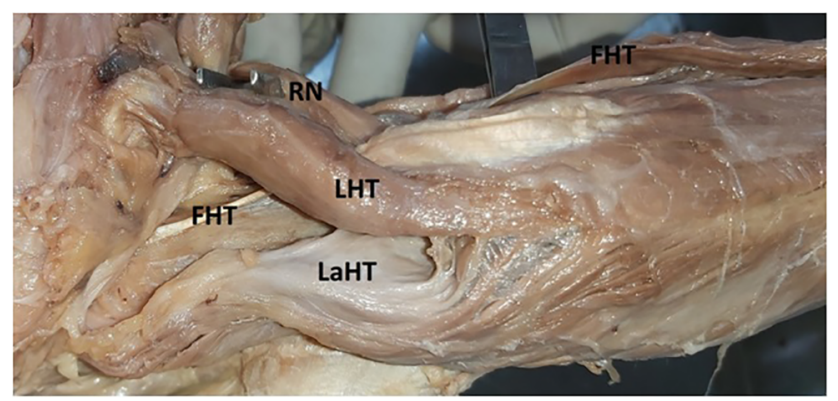

Fig. 1 Fourth head of triceps brachii muscle. FHT, fourth head of triceps; LaHT, lateral head of triceps; LHT, long head of triceps; RN, radial nerve.

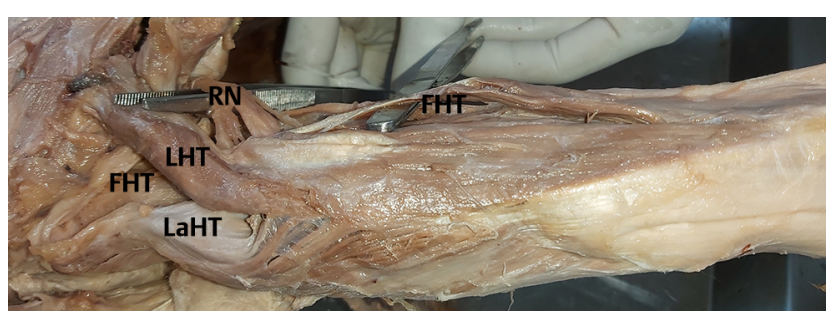

Fig. 2 The nerve supply and insertion of fourth head of triceps brachii. FHT: fourth head of triceps; LaHT, lateral head of triceps; LHT, long head of triceps; RN, radial nerve. belly on the distal one-third of the humerus. ${ }^{3}$ Similarly, ipsilateral four-headed triceps brachii muscle was found by Tubbs et al. ${ }^{4}$ This head originated from the posterior aspect of the surgical neck of the humerus.

Cheema and Singla also found extra head of triceps brachii muscle originating from humerus from the medial side of surgical neck of humerus. The muscle belly of extra head was blending with the long head of triceps brachii muscle. ${ }^{5}$ Kulkarni et al found the fourth head of triceps brachii that was arising from the upper part of the posterior aspect of the shaft of the humerus immediately below the capsule of the shoulder joint and from the upper part of the oblique line along with lateral head of triceps brachii. The origin was by a long tendon which extended on the medial aspect of arm after crossing the radial nerve and profunda brachii vessels superficially along with the lateral head of triceps brachii. In the lower one-third of the posterior aspect of the arm, the tendon continued as muscular belly which merged with the medial part of medial head of triceps brachii. ${ }^{6}$

Khona and Ashwini in their study on 60 upper limbs found fourth head of origin of muscle observed in two different male cadavers in right and left arms, respectively. The variations present were seen only unilaterally in both cadavers. One of the variations of fourth head was similar to the present study with tendinous origin from posteromedial aspect of upper part of the shaft of humerus and running over the neurovascular bundle, containing radial nerve and profunda brachii artery. The second variation was taking its origin from the posterior part of the capsule of the shoulder joint and the fourth head was muscular throughout and no tendon was found.

\section{Clinical Relevance of the Variation}

Clinically, the proximity of the tendon of the fourth head to the radial nerve and the deep brachial artery may be a contributing factor for their compression, especially during a strenuous exertion or muscular contraction, resulting in a neurovascular compromise. Thus, clinicians diagnosing or treating patients with weakness or pain of posterior arm should consider muscular variations of this compartment that may result in neurovascular compression. The variant triceps muscle is clinically important for surgeons dealing with entrapment or compressive neuropathies, orthopaedicians operating on fractures of the humerus, anesthetists performing pain management therapies on the upper limb, radiologists doing radiodiagnostic procedures, for example, CT scan, MRI of the arm and angiographic studies, and physiotherapists doing electromyography for evaluating and recording electric activity produced by the skeletal muscles. ${ }^{8}$

\section{Embryological Basis}

From an embryological aspect, the lateral plate mesoderm migrates into the upper limb bud and condenses along the central axis to form the skeletal components. The mesoderm from the somites migrates into the limb bud and condenses to form the muscle component of the upper limb. During week 5, the mesoderm forms these somites (myotomes) and gives rise to a posterior and anterior condensation. 
The mesoderm of these two condensations differentiates into myoblasts. The triceps brachii muscle is derived from the posterior condensation and it is possible that during this period, such accessory muscles or muscle heads can originate. ${ }^{9}$ Also in the present study, neurovascular bundles might have separated the fourth head from the medial head and long head of triceps brachii, respectively.

\section{Comparative Anatomy}

Comparative anatomically, the triceps brachii muscle in carnivores is bulky and is made up of many sub heads of the muscle, long head has anterior and posterior parts. Branches of the profunda brachii artery and vein pass out between these two parts, medial head has small-to-intermediate and long heads, and lateral head

According to this, the present variation in the study may be due to nonfusion of these heads during evolution.

\section{Conclusion}

Though the anatomical variations of the triceps brachii muscle are fairly rare, they can be of importance for clinicians who perform surgical intervention on the upper limb. Thus, surgeons, anesthetists, orthopaedicians, radiologists, and also anatomists should have knowledge about the incidence of such variations. The probable reason for the presence of extra heads of triceps brachii may be due to interference in the development of musculature of upper limb by any connective tissue or neurovascular bundle or during evolution, the fusion or disappearance of the subheads of the three heads of the triceps brachii muscle is not completed.

\section{Conflict of Interest}

None declared.

\section{References}

1 Standring S, Gray's Anatomy: The Anatomical Basis of Clinical Practice. 40th ed. London, United Kingdom: Churchill Livingstone Elsevier; 2008

2 Anson BJ, Morris' Human Anatomy. 12th ed. New York, NY: McGraw-Hill; 1966

3 Fabrizio PA, Clemente FR. Variation in the triceps brachii muscle: a fourth muscular head. Clin Anat 1997;10(4):259-263

4 Tubbs RS, Salter EG, Oakes WJ. Triceps brachii muscle demonstrating a fourth head. Clin Anat 2006;19(7):657-660

5 Cheema P, Singla R. Four headed triceps brachii muscle. Int J Anat Var 2011;4:43-44

6 Kulkarni R, Kulkarni RN, Solan S. Unilateral right quadriceps brachii - a case report. Anatomica Karnataka 2012;6:15-17

7 Khona P, Ashwini C. A study of variations of the triceps brachii muscle in north Karnataka population. Indian Journal of Anatomy \& Surgery of Head. Neck \& Brain 2017;3(4):101-103

8 Iliev AL, Dandov A, Jelev L, Hinova-Palova D. An aberrant fourth head of the triceps brachii muscle. PRAEMEDICUS 2015;30(1):9-12

9 Schoenwolf GC, Bleyl SB, Brauer PR, Francis-West PH, Larsen's Human Embryology. 4th ed. Philadelphia, PA: Churchill Livingstone; 2009: 241-242 